BMJ

Open

Gastroenterology

\title{
Weight regain after Roux-en-Y gastric bypass has a large negative impact on the Bariatric Quality of Life Index
}

\author{
Pichamol Jirapinyo, ${ }^{1,2}$ Barham K Abu Dayyeh, ${ }^{3}$ Christopher C Thompson ${ }^{1,2}$
}

To cite: Jirapinyo $P, A b u$ Dayyeh BK, Thompson CC Weight regain after Rouxen- $Y$ gastric bypass has a large negative impact on the Bariatric Quality of Life Index. BMJ Open Gastro 2017;4:e00153. doi:10.1136/ bmjgast-2017-000153

- Additional material is published online only. To view please visit the journal online (http://dx.doi.org/10.1136/ bmjgast-2017-000153)

Received 14 May 2017 Revised 6 July 2017 Accepted 7 July 2017
Check for updates

${ }^{1}$ Division of Gastroenterology, Hepatology and Endoscopy, Brigham and Women's Hospital, Boston, Massachusetts, USA ${ }^{2}$ Harvard Medical School, Boston, Massachusetts, USA ${ }^{3}$ Division of Gastroenterology and Hepatology, Mayo Clinic, Rochester, Minnesota, USA

Correspondence to Dr Christopher C Thompson; cthompson@hms.harvard.edu

\section{ABSTRACT}

Background Despite initial successful weight loss, some patients may experience weight regain following Rouxen-Y gastric bypass (RYGB).

Objective To assess the impact of weight regain on bariatric patients' quality of life (QoL).

Methods This was a prospective cross-sectional study. Fifty-six consecutive RYGB patients were recruited and divided into weight-regain and weight-stable cohorts. QoL was assessed using the Bariatric Quality of Life (BQL) questionnaire. The BQL Index scores of the weightregain and weight-stable groups were compared using Student's $t$-test. Additionally, the BQL Index score of the weight-regain group was compared with that of historical prebariatric patients. Predictors of BQL were assessed using univariate and multivariate linear regression analyses.

Results 0 56 RYGB patients, 41 (73\%) had weight regain. On average, patients had body mass index (BMI) of $37 \pm 7.5 \mathrm{~kg} / \mathrm{m}^{2}$ and gained $34 \pm 26 \%$ of maximal weight initially lost. Weight-regain patients had lower BQL Index scores than weight-stable patients ( $44.8 \pm 6$ vs $53 \pm 7$, $\mathrm{p}<0.001)$. Patients with weight regain had similar $\mathrm{BQL}$ Index scores as the prebariatric patients despite lower BMI (BMI of $39.7 \pm 6.8$ vs $47.2 \pm 7.6, p<0.05$; BQL Index of $44.8 \pm 6$ vs $41.6 \pm 10.4, p=0.144$, respectively). Years from RYGB, BMI and amount of weight regain were associated with BQL Index on a univariate analysis $(\beta=-0.55,-0.52$, $-0.7 ; p<0.0001)$. Only weight regain was a significant predictor of $B Q L$ on a multivariate analysis $(\beta=-0.56$; $\mathrm{p}=0.001$ ).

Conclusion Weight regain had a negative impact on bariatric patients' QoL. Patients who regained at least 15\% of maximal weight lost appeared to have as low QoL as those who had not undergone bariatric surgery despite a lower BMl.

\section{INTRODUCTION}

Roux-en-Y gastric bypass (RYGB) is a commonly performed bariatric surgery. ${ }^{1-3}$ Although RYGB is an effective procedure, it has been estimated that $15 \%-35 \%$ of patients will not achieve satisfactory weight loss ( $\geq 50 \%$ excess weight loss) or will experience significant weight regain (gaining of $\geq 15 \%$ of the maximal weight initially lost) ${ }^{3-9}$ Gastroenterologists are caring for ever increasing
Summary box

What is already known about this subject?

- Weight regain is not infrequent after Roux-en-Y gastric bypass (RYGB).

- Bariatric Quality of Life (BQL) questionnaire is a validated and objective survey that assesses quality of life (QoL) of bariatric patients before and after surgery.

- Weight regain following pharmacotherapy for obesity is associated with worsening QoL, although not back to baseline.

What are the new findings?

- Patients who experience weight regain following RYGB have worse QoL than those whose weights remain stable after surgery.

- Patients who experience weight regain following RYGB have similar QoL as those who have not undergone RYGB despite lower body mass index (BMI).

- Weight regain is a significant negative predictor of QoL after bariatric surgery after controlling for age, $\mathrm{BMI}$ and years from RYGB.

How might this impact on clinical practice in the foreseeable future?

- Understanding the negative impact that weight regain has on QoL highlights the importance of close follow-up and its early recognition.

- More intensive multidisciplinary care to prevent weight regain is critical to improve long-term success of bariatric surgery.

- Gastroenterologists should become familiar with pharmacological and endoscopic treatment options to manage weight regain.

numbers of patients with RYGB, and there are now many endoscopic options available to treat weight regain.

Traditionally, weight loss and resolution of obesity-related comorbid illnesses have been used as primary outcomes for bariatric surgeries. However, of equal importance is patients' quality of life (QoL), which sometimes may be considered as a true measure of effectiveness of a surgical procedure. In 2005 , 


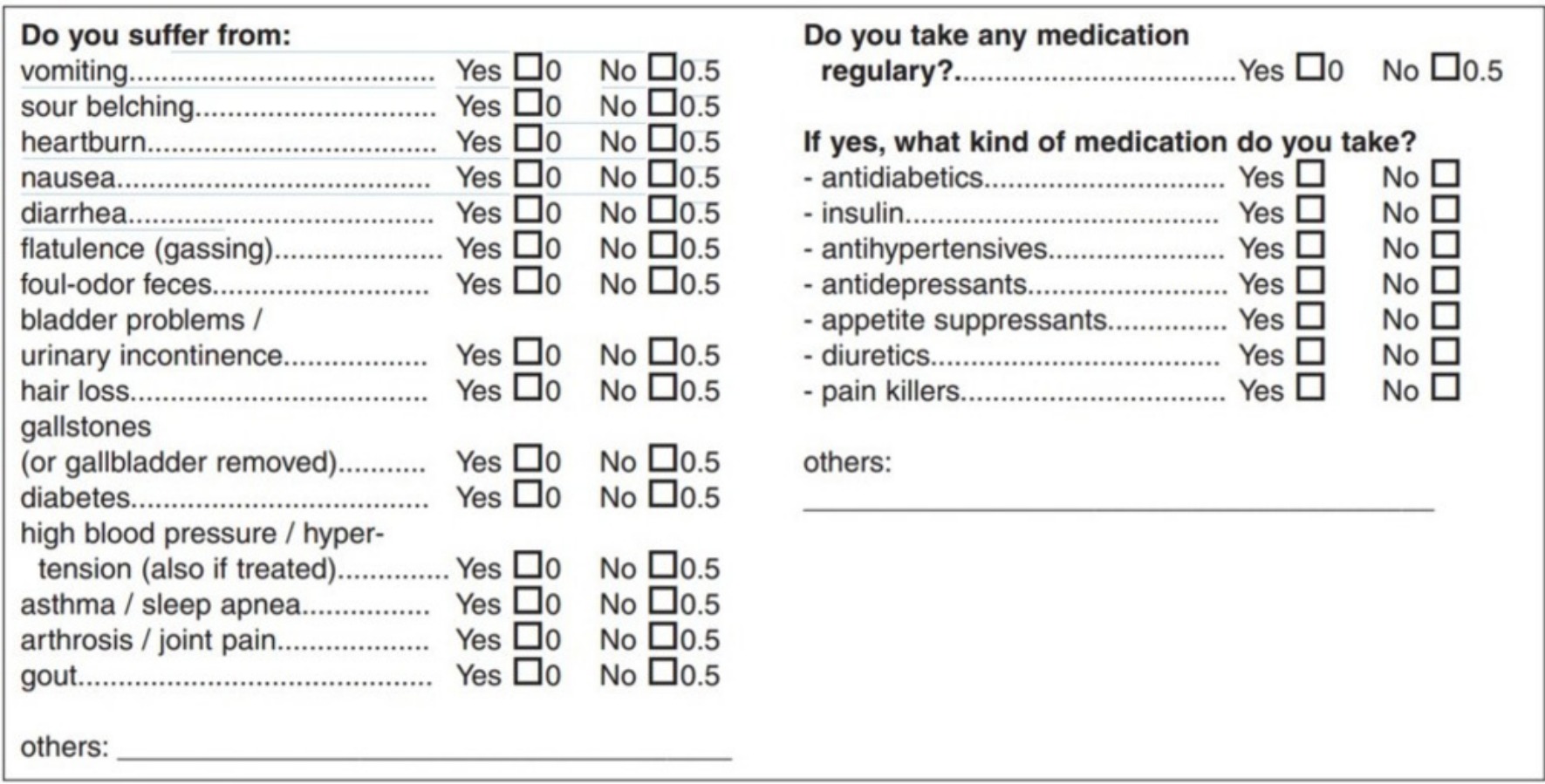

Figure 1 The Bariatric Quality of Life questionnaire (part 1). From Weiner et al. ${ }^{18}$

Weiner and colleagues developed the Bariatric Quality of Life (BQL) questionnaire, which was a validated and objective survey that assessed QoL of bariatric patients before and after the surgery. It consisted of questions related to weight, obesity-related comorbid illnesses, surgery-related gastrointestinal symptoms, psychological well-being, social function and physical functioning. The questionnaire has been demonstrated to be sensitive to changes after bariatric surgical interventions, and therefore has been used to assess the effectiveness of multiple bariatric and metabolic surgical procedures. ${ }^{10-12}$ While it has been previously demonstrated that RYGB is associated with improved QoL, ${ }^{13} 14$ the effect of weight regain after initial weight loss following bariatric surgery on patients' life quality remains unknown.

A previous study of pharmacological therapy for obesity demonstrated that QoL improved with initial weight loss and subsequently worsened with weight regain. However, the QoL with weight regain remained better than that of the pretreatment assessment. ${ }^{15}$ As such, we hypothesised that the BQL Index scores would be lower in weight-regain patients than those in weight-stable patients, yet higher than the pretreatment cohort.

This study aimed to assess the impact of weight regain on bariatric patient's QoL. Additionally, predictors of post-BQL were determined.

\section{MATERIALS AND METHODS \\ Study design}

This study was a prospective cross-sectional study that was conducted at the Brigham and Women's Hospital (Boston, Massachusetts, USA) Fifty-six consecutive RYGB patients were enrolled at the weekly bariatric centre of excellence at the Department of Surgery. Inclusion criteria was a history of RYGB of at least 1 year prior to the time of study enrolment. Exclusion criteria included a history of revisional surgery, transoral outlet reduction (TORe) or other endoscopic intervention for weight regain. Patients' demographics, weight profile, height and comorbidities were collected. The BQL questionnaire was then administered. All participants provided written informed consents. The study was approved by the institution review board committee.

\section{Definitions}

Body mass index (BMI) was calculated using the formula: weight in kilograms/(height in metres) ${ }^{2}$. Amount of weight regain was calculated using the formula (current weight-nadir weight) / (pre-RYGB weight - nadir weight) $\mathrm{x} 100 \%$. In this study, weight regain was defined as gaining of at least $15 \%$ of the weight that was initially lost after bariatric surgery. This was based on available literature that defined weight regain as gaining of $5 \%$ to $30 \%$ or more of maximal weight initially lost. ${ }^{16}{ }^{17}$ Stable weight was defined as gaining of $<15 \%$ of maximal weight initially lost.

\section{Part I: Association between weight regain and BQL}

The RYGB cohort was divided into two groups: those with weight regain and those with stable weight. The BQL Index scores of the weight-regain and weight-stable groups were compared. Additionally, the BQL Index scores of the weight-regain group were also compared with those of the historical prebariatric surgery patients. Specifically, this control group consisted of patients with obesity (BMI $\geq 30 \mathrm{~kg} / \mathrm{m}^{2}$ ), whose QoL were assessed using BQL questionnaire prior to undergoing bariatric surgery. 


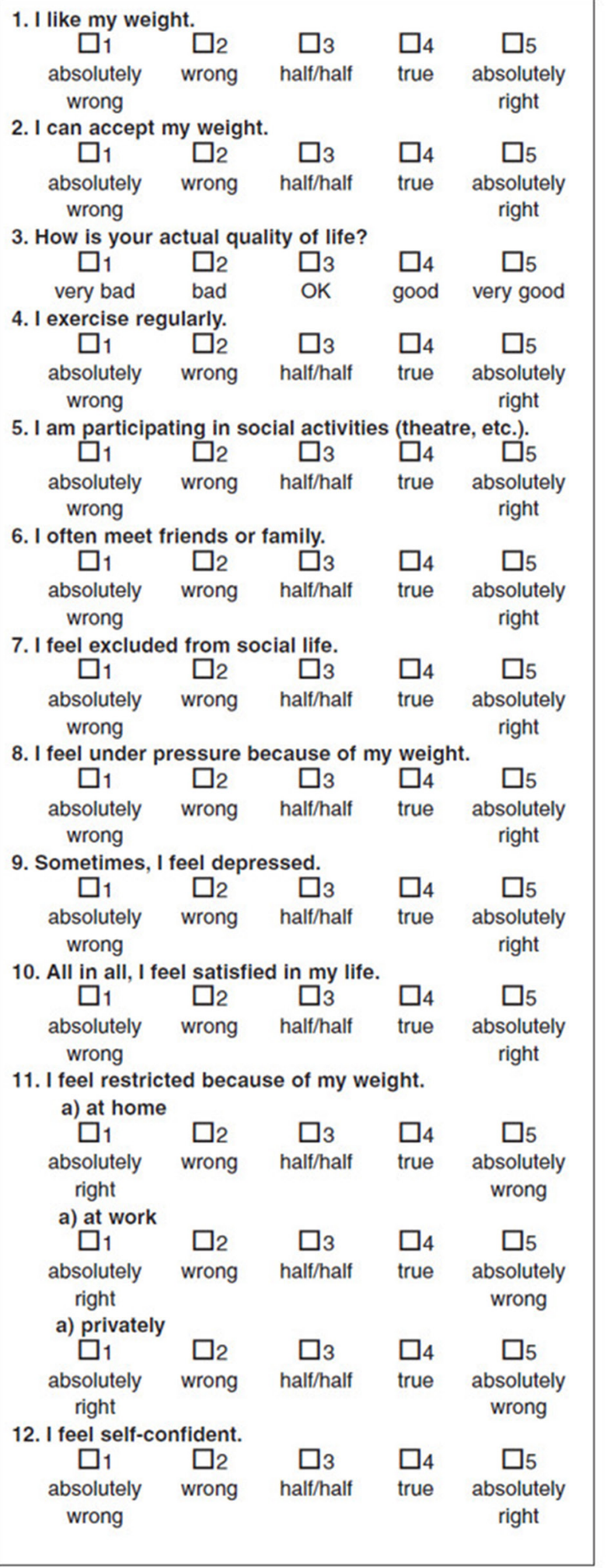

Figure 2 The Bariatric Quality of Life questionnaire (part 2). From Weiner et al $^{18}$

This was previously collected as part of a separate study protocol. ${ }^{18} 19$

\section{Part Il: Predictors of BQL}

Predicting factors of QoL after RYGB were determined using univariate and multivariate linear regression.
Potential predictors, which were included in the model, were age, years from RYGB, BMI and the amount of weight regain.

\section{BQL questionnaire}

The BQL questionnaire is a validated instrument that was designed to assess patients' QoL after bariatric procedures. ${ }^{18}{ }^{19}$ It consists of 30 questions divided into two sections. The first section consisting of 16 items assesses non-QoL factors including obesity-related comorbidities, gastrointestinal symptoms that are common after bariatric surgery and medication intake (figure 1). The second section consisting of 14 items assesses QoL factors with a five-point Likert scale ranging from 0 to 5 points (figure 2). The BQL Index score is calculated by adding all the item scores from both sections, and ranges from a scale of 0 to 78 with a higher score representing better QoL.

\section{Statistical analysis}

All continuous variables were expressed as mean $\pm \mathrm{SD}$ deviation. Categorical variables were expressed as proportions $(\%)$. Student's $t$-test was used to compare continuous measures between the two groups, and Pearson's $\mathrm{X}^{2}$ test was used for comparison of categorical variables. Univariate linear regression analysis was used to determine an association between BQL Index scores and age, years from RYGB, BMI and the amount weight regain. Multivariate linear regression analysis was then performed to adjust for potential confounders of BQL Index scores. Standardised $\beta$ coefficients were reported and a significant two-sided $p$ value was set at 0.05 or less. All statistical modelling was performed using SAS V. 9.2 software (Cary, North Carolina, USA). Graphing was done using the JMP V. 8 software (SAS Institute).

Power analysis for two means was conducted using SAS Power and Sample Size application to determine a sufficient sample size for an $8 \%$ difference in BQL Index scores between the prebariatric control group and the weight-regain group, with $\alpha$ of 0.05 and power of 0.80 . Since there have been no studies evaluating the effect of weight regain after bariatric surgery on the change in QoL, we estimated that this effect based on a previous prospective cohort study of patients with weight regain after pharmacotherapy and lifestyle therapy. ${ }^{15}$ Specifically, this previous study showed that patients who regained weight after initial weight loss with pharmacotherapy and lifestyle therapy had a lower health-related quality of life (HRQOL) score than that at the nadir weight by $4.8 \%$, and a higher HRQOL score than their baseline HRQOL score by $8.4 \%$. Based on the aforementioned assumptions, the desired sample size was 36 .

\section{RESULTS}

Part I: Association between weight regain and BQL

A total of 56 consecutive RYGB patients were enrolled in the study. Of these, $41(73 \%)$ had weight regain. Baseline characteristics of the weight-regain and weight-stable 
Table 1 Baseline characteristics

\begin{tabular}{|c|c|c|c|c|c|}
\hline & $\begin{array}{l}\text { Weight-regain } \\
\text { cohort }(n=41)\end{array}$ & $\begin{array}{l}\text { Weight-stable } \\
\text { cohort }(n=15)\end{array}$ & $\begin{array}{l}\text { Presurgical obese } \\
\text { control }(n=133)\end{array}$ & p Value* & p Value ${ }^{\dagger}$ \\
\hline Age (years) mean $\pm S D$ & $49 \pm 9.5$ & $49 \pm 11$ & $37.6 \pm 12$ & 1.00 & $<0.0001$ \\
\hline Female (\%) & 93 & 87 & 81 & 0.49 & 0.08 \\
\hline BMI $\left(\mathrm{kg} / \mathrm{m}^{2}\right)$ mean $\pm S D$ & $39.7 \pm 6.8$ & $31 \pm 5.8$ & $47.2 \pm 7.6$ & $<0.0001$ & $<0.0001$ \\
\hline Years from RYGB (years) (mean \pm SD) & $7.0 \pm 3.8$ & $2.2 \pm 0.9$ & - & $<0.0001$ & - \\
\hline Weight regain (\%) mean \pm SD & $44.7 \pm 4.8$ & $4.5 \pm 4.8$ & - & $<0.0001$ & - \\
\hline
\end{tabular}

${ }^{*}$ Comparison between the weight-regain cohort and weight-stable cohort.

†Comparison between the weight-regain cohort and the presurgical obese control.

BMI, body mass index; RYGB, Roux-en-Y gastric bypass.

cohorts are shown in table 1. Specifically, age and sex distributions were similar between the weight-regain and weight-stable cohorts. However, the weight-regain group had a higher BMI and had a greater time duration from RYGB compared with the weight-stable group at the time of BQL assessment. When compared with the prebariatric control group, the weight-regain group appeared to be older and had lower BMI (table 1). Additionally, patients were assessed by year from RYGB as shown in figure 3 .

The BQL Index scores of the weight-regain, weightstable and prebariatric groups are shown in table 2. Overall, the weight-regain group had significantly lower BQL Index scores than those of the weight-stable group $(\mathrm{p}<0.001)$. When compared with the prebariatric control, the weight-regain group had similar BQL Index scores $(\mathrm{p}=0.144)$ despite having a lower BMI $(39.7 \pm 6.8 \mathrm{vs}$ $47.2 \pm 7.6$, respectively).

\section{Part II: Predictors of BQL}

On a univariate linear regression analysis, years from RYGB, BMI and weight regain were associated with BQL Index scores $(\beta=-0.55,-0.52,-0.7$, respectively; $\mathrm{p}<0.0001$ for all). Age was not a significant predictor of BQL Index scores. On a multivariate linear regression, only weight regain remained a significant predictor of BQL Index scores $(\beta=-0.56 ; \mathrm{p}=0.001)$ after controlling

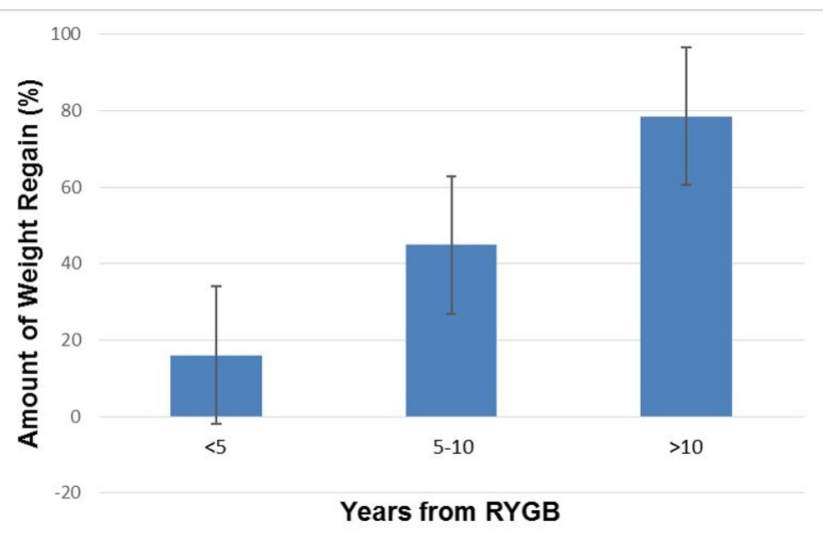

Figure 3 Amount of weight regain in patients who were $<5$, $5-10$ and $>10$ years post-RYGB. RYGB, Roux-en-Y gastric bypass. for age, years from RYGB and BMI (table 3). Years from RYGB and BMI were no longer associated with BQL Index scores after controlling for potential confounders. As demonstrated in figure 4 , the association between weight regain and BQL Index scores was of a linear relationship with a negative Pearson correlation coefficient, which suggested that a greater amount of weight regain was associated with a lower BQL Index score.

\section{DISCUSSION}

Weight regain is not infrequent after RYGB and may be associated with recurrence of presurgical comorbid conditions. ${ }^{20}$ In this study, we demonstrate that in addition to a return of comorbid illnesses, weight regain is also associated with worsening QoL. Specifically, the study shows that bariatric patients who regain at least $15 \%$ of the maximal weight lost seem to have similar QoL as patients with morbid obesity who have not undergone bariatric surgery despite an overall weight loss and a lower BMI. Additionally, our study also shows that the amount of weight regain is negatively and linearly correlated with postbariatric QoL.

This study is the first to demonstrate an association between weight regain following RYGB and Health Related Quality of Life (HRQL). Previous studies have demonstrated that RYGB led to an improvement in QoL. However, none have clarified the impact of weight regain. In Schauer et al, HRQL was reported as one of the secondary outcomes. In this study, patients in the RYGB group had a significantly higher HRQL compared with those in the medical-therapy group. ${ }^{13}$ Similarly, the Swedish Obese Subject study demonstrated that HRQL improved after RYGB and peaked at 0.5 to 1 year. Subsequently, HRQL declined, which was hypothesised to be related to weight regain. ${ }^{14}$ This concept had remained to be proven.

Our study emphasises the importance of frequent assessment of QoL after bariatric surgery, or any weight loss intervention. Compared with other reported outcomes such as weight loss or resolution of obesity-related comorbid illnesses, QoL has usually been underused in both clinical and research settings. As demonstrated in the first part of our study, QoL of the weight-regain group 
Table 2 Bariatric Quality of Life (BQL) Index scores

\begin{tabular}{llllll} 
& $\begin{array}{l}\text { Weight-regain } \\
\text { cohort }(\mathbf{n}=\mathbf{4 1})\end{array}$ & $\begin{array}{l}\text { Weight-stable } \\
\text { cohort }(\mathbf{n}=15)\end{array}$ & $\begin{array}{l}\text { Presurgical obese } \\
\text { control }(\mathbf{n}=133)\end{array}$ & $\mathbf{p ~ V a l u e}^{*}$ & $\mathbf{p ~ V a l u e ~}^{\dagger}$ \\
\hline BQL Index score mean \pm SD & $44.8 \pm 6$ & $53 \pm 7$ & $41.6 \pm 10.4$ & $<0.001$ & 0.144 \\
\hline
\end{tabular}

${ }^{*}$ Comparison between the weight-regain cohort and weight-stable cohort.

†Comparison between the weight-regain cohort and the presurgical obese control.

with a BMI of $39.7 \pm 6.8$ is as low as that of the prebariatric

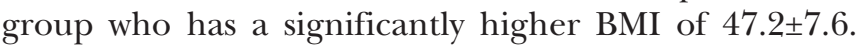
If BMI or a change in BMI alone was the only reported outcome, one might not have detected the negative impact that the bariatric surgery, specifically weight regain following bariatric surgery, had on patients' life.

By demonstrating that weight regain is associated with worsening QoL, this study also highlights the importance of early recognition and prompt response to weight regain. As mentioned earlier, weight regain is a common complication after RYGB. It is approximated that $30 \%-35 \%$ of lost weight is regained after 1 year following bariatric surgery and that $50 \%$ of patients will return to their baseline weight after 5 years. ${ }^{21}$ Despite its prevalence, exact aetiologies of weight regain remain unclear. Behavioural, medical and anatomical factors have been proposed to play a role. ${ }^{2223}$ Behavioural factors may include nutritional habits and psychological factors. Possible medical factors include hypothyroidism, anaemia, genetics, microbiota and orthopaedic status. From an anatomical standpoint, the presence of a gastrogastric fistula and dilated gastrojejunal aperture (GJA) has previously been demonstrated to be associated with weight regain. ${ }^{24-26}$ It is important to address all behavioural and medical causes prior to considering procedural interventions. Some surgical and endoscopic techniques developed to treat weight regain focus on treating these anatomical factors. While surgical revision has traditionally been used to treat weight regain, the procedure is associated with limited efficacy data and higher complication rates than that of the index surgery. ${ }^{27-30}$ Alternatively, endoscopic techniques have recently been developed to treat weight regain. These include sclerotherapy where sodium morrhuate is injected around the GJA to reduce the GJA size, ${ }^{31} 32$ argon plasma coagulation, ${ }^{33} 34$ endoscopic plication and endoscopic suturing to close a small gastrogastric fistula and/or to reduce the size of the GJA. ${ }^{35-39}$ A recent multicentre, randomised, blinded, sham-controlled trial has provided level I evidence to support the use of sutured TORe procedure to treat weight regain following RYGB. In this study, a superficial suction-based suturing device was used. ${ }^{40}$ A more recent study has shown that a new full-thickness suturing device provides a greater and more durable weight loss compared with the device studied in the randomised controlled trial. ${ }^{41}$ Furthermore, longer term data with 3-year follow-up has confirmed the durability of this procedure. ${ }^{42}$

In addition to identifying and intervening on the anatomical risk factors for weight regain, it is equally important to pay attention to and provide support for behavioural and psychological aspects of weight regain. ${ }^{43}$ This is best accomplished via a multidisciplinary approach, where bariatric surgeons, gastroenterologists, endocrinologists, psychiatrists, dieticians and physical therapists all work together to directly address the effect that weight regain may have on QoL in order to maximise the likelihood of successful outcomes.

One limitation of this study is the lack of established definition of weight regain in the current literature. ${ }^{44}$ Our study uses a cut-off of $15 \%$ or greater as the definition of significant weight regain. This is based on literature review, which reveals that previous studies have defined weight regain as gaining of a range of $5 \%$ to $30 \%$ or greater of maximal weight initially lost. ${ }^{16}{ }^{17}$ With the cut-off of $15 \%$ or greater, our study is able to show the difference in BQL Index scores between those with and without weight regain and demonstrate an association between weight regain and QoL regardless of age, BMI and years from RYGB. This suggests that a gain of at least $15 \%$ of maximal weight loss is clinically significant. A prospective study to further evaluate the effect of different levels of weight regain on QoL and health metrics is needed. Additionally, in our study, $73 \%$ of the RYGB patients consecutively recruited had weight regain. This number was higher than the prevalence of weight regain reported in previous studies. This could be due

\begin{tabular}{|c|c|c|c|c|}
\hline & \multicolumn{2}{|l|}{ Univariate } & \multicolumn{2}{|l|}{ Multivariate } \\
\hline & Standardised $\beta$ coefficient & p-value & Standardised $\beta$ coefficient & p Value \\
\hline Age & 0.054 & 0.71 & -0.16 & 0.16 \\
\hline Years from RYGB & -0.55 & $<0.0001$ & -0.09 & 0.60 \\
\hline BMI & -0.52 & $<0.0001$ & -0.20 & 0.15 \\
\hline Weight regain & -0.7 & $<0.0001$ & -0.56 & 0.001 \\
\hline
\end{tabular}

BMI, body mass index; BQL, Bariatric Quality of Life; RYGB, Roux-en-Y gastric bypass. 


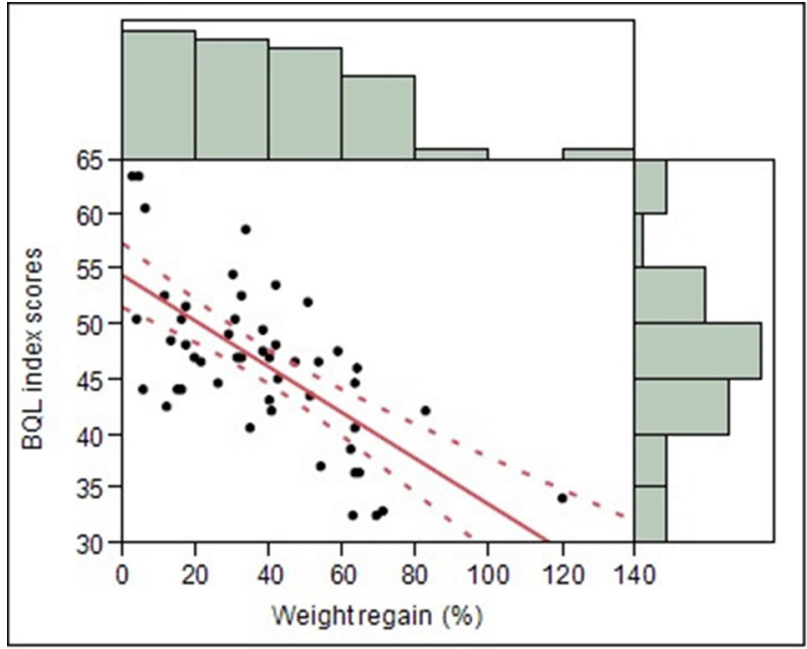

Figure 4 Scatter plot with best-fit linear regression line and $95 \% \mathrm{Cl}$ of the association between weight regain and Bariatric Quality of Life (BQL) Index scores.

to the retrospective nature of the study, and the fact that those with weight regain were more likely to seek medical care at a later date. Additionally, this was in part due to the revisional procedure expertise of one surgeon in the Bariatric Centre of Excellence. Furthermore, the number of patients within each group-the weight-regain $(n=41)$, weight-stable $(n=15)$ and prebariatric surgery $(n=133)$ groups-varied. Nevertheless, the study is powered to detect a difference in QoL levels between the weight-regain and prebariatric surgery groups, based on a previous study showing the change in QoL of patients who regained weight after pharmacotherapy and lifestyle intervention.

With a rise in the obesity pandemic, the number of bariatric surgeries being performed and their complications including weight regain will continue to increase. Understanding the negative impact that weight regain has on bariatric patients' QoL highlights the importance of close and long-term follow-up postbariatric surgery. Additionally, early recognition of weight regain is essential and a multidisciplinary approach should be administered to address weight regain in order to optimise postbariatric care. Gastroenterologists should become more familiar with endoscopic treatment options to better help this patient population.

Acknowledgements The authors would like to thank Dr David B Lautz for providing patients and study guidance.

Contributors PJ: data collection and analysis; drafting of the manuscript. BKAD: data collection and analysis. CT: study design, critical revision of the manuscript.

Funding The project was supported by the Clinical Translational Science Award from the National Center for Research Resources (UL1RR025758) and the Harvard Digestive Diseases Center at Harvard Medical School (DK034854).

Competing interests None declared.

Ethics approval Institutional review board.

Provenance and peer review Not commissioned; externally peer reviewed.
Data sharing statement Inquiries regarding access to the data should be addressed to the corresponding author and will be reviewed by the institutional review board.

Open Access This is an Open Access article distributed in accordance with the Creative Commons Attribution Non Commercial (CC BY-NC 4.0) license, which permits others to distribute, remix, adapt, build upon this work non-commercially, and license their derivative works on different terms, provided the original work is properly cited and the use is non-commercial. See: http://creativecommons.org/ licenses/by-nc/4.0/

(c) Article author(s) (or their employer(s) unless otherwise stated in the text of the article) 2017. All rights reserved. No commercial use is permitted unless otherwise expressly granted.

\section{REFERENCES}

1. Buchwald H, Oien DM. Metabolic/bariatric surgery worldwide 2011. Obes Surg 2013;23:427-36.

2. Khorgami Z, Shoar S, Andalib A, et al. Trends in utilization of bariatric surgery, 2010-2014: sleeve gastrectomy dominates. Surg Obes Relat Dis 2017;13:774-8.

3. Ponce J, Nguyen NT, Hutter M, et al. American Society for Metabolic and Bariatric Surgery estimation of bariatric surgery procedures in the United States, 2011-2014. Surg Obes Relat Dis 2015:11:1199-200.

4. Dakin GF, Eid G, Mikami D, et al. Endoluminal revision of gastric bypass for weight regain--a systematic review. Surg Obes Relat Dis 2013;9:335-42.

5. Brolin RE. Bariatric surgery and long-term control of morbid obesity. JAMA 2002;288:2793-6.

6. Elder KA, Wolfe BM. Bariatric surgery: a review of procedures and outcomes. Gastroenterology 2007;132:2253-71.

7. Magro DO, Geloneze B, Delfini R, et al. Long-term weight regain after gastric bypass: a 5-year prospective study. Obes Surg 2008:18:648-51.

8. Christou NV, Look D, Maclean LD. Weight gain after short- and longlimb gastric bypass in patients followed for longer than 10 years. Ann Surg 2006;244:734-40.

9. Yimcharoen $\mathrm{P}$, Heneghan HM, Singh M, et al. Endoscopic findings and outcomes of revisional procedures for patients with weight recidivism after gastric bypass. Surg Endosc 2011;25:3345-52.

10. Buchwald $\mathrm{H}$, Avidor $\mathrm{Y}$, Braunwald $\mathrm{E}$, et al. Bariatric surgery: a systematic review and meta-analysis. JAMA 2004;292:1724-37.

11. Kolotkin RL, Crosby RD, Williams GR. Assessing weight-related quality of life in obese persons with type 2 diabetes. Diabetes Res Clin Pract 2003:61:125-32.

12. Vasiljevic N, Ralevic S, Kolotkin RL, et al. The relationship between weight loss and health-related quality of life in a serbian population. Eur Eat Disord Rev 2012;20:162-8.

13. Schauer PR, Bhatt DL, Kirwan JP, et al. Bariatric surgery versus intensive medical therapy for diabetes--3-year outcomes. $N$ Engl $J$ Med 2014;370:2002-13.

14. Karlsson J, Taft C, Rydén A, et al. Ten-year trends in health-related quality of life after surgical and conventional treatment for severe obesity: the SOS intervention study. Int J Obes 2007;31:1248-61.

15. Engel SG, Crosby RD, Kolotkin RL, et al. Impact of weight loss and regain on quality of life: mirror image or differential effect? Obes Res 2003;11:1207-13.

16. Tamboli RA, Breitman I, Marks-Shulman PA, et al. Early weight regain after gastric bypass does not affect insulin sensitivity but is associated with elevated ghrelin. Obesity 2014;22:1617-22.

17. DiGiorgi M. Factors associated with long term weight regain after bariatric surgery (doctoral dissertation). 2012. file:///C:/Users/Pichamol\%20Jirapinyo/Downloads/DiGiorgi_ columbia_0054D_10784\%20(1).pdf (accessed 28 Mar 2017).

18. Weiner S, Sauerland S, Fein M, et al. The Bariatric Quality of Life index: a measure of well-being in obesity surgery patients. Obes Surg 2005; 15:538-45.

19. Weiner S, Sauerland S, Weiner R, et al. Validation of the adapted Bariatric Quality of Life Index (BQL) in a prospective study in 446 bariatric patients as one-factor model. Obes Facts 2009;2:63-6.

20. Shah M, Simha V, Garg A. Review: long-term impact of bariatric surgery on body weight, comorbidities, and nutritional status. J Clin Endocrinol Metab 2006;91:4223-31.

21. Blomain ES, Dirhan DA, Valentino MA, et al. Mechanisms of weight regain following weight loss. ISRN Obes 2013;2013:1-7.

22. Maleckas A, Gudaityte R, Petereit $\mathrm{R}$, et al. Weight regain after gastric bypass: etiology and treatment options. Gland Surg 2016;5:617-24. 
23. Freire $\mathrm{RH}$, Borges $\mathrm{MC}$, Alvarez-Leite Jl, et al. Food quality, physical activity, and nutritional follow-up as determinant of weight regain after Roux-en-Y gastric bypass. Nutrition 2012;28:53-8.

24. Abu Dayyeh BK, Lautz DB, Thompson CC. Gastrojejunal stoma diameter predicts weight regain after Roux-en-Y gastric bypass. Clin Gastroenterol Hepatol 2011;9:228-33.

25. Heneghan HM, Yimcharoen P, Brethauer SA, et al. Influence of pouch and stoma size on weight loss after gastric bypass. Surg Obes Relat Dis 2012;8:408-15.

26. O'Brien CS, Wang G, McGinty J, et al. Effects of gastrogastric fistula repair on weight loss and gut hormone levels. Obes Surg 2013;23:1294-301.

27. Vijgen GH, Schouten R, Bouvy ND, et al. Salvage banding for failed Roux-en-Y gastric bypass. Surg Obes Relat Dis 2012;8:803-8.

28. Yimcharoen $\mathrm{P}$, Heneghan $\mathrm{H}$, Chand $\mathrm{B}$, et al. Successful management of gastrojejunal strictures after gastric bypass: is timing important? Surg Obes Relat Dis 2012;8:151-7.

29. Rawlins ML, Teel D, Hedgcorth $\mathrm{K}$, et al. Revision of Roux-en-Y gastric bypass to distal bypass for failed weight loss. Surg Obes Relat Dis 2011;7:45-9.

30. Brethauer SA, Kothari S, Sudan R, et al. Systematic review on reoperative bariatric surgery: American Society for Metabolic and Bariatric Surgery Revision Task Force. Surg Obes Relat Dis 2014;10:952-72.

31. Abu Dayyeh BK, Jirapinyo P, Weitzner Z, et al. Endoscopic sclerotherapy for the treatment of weight regain after Roux-en-Y gastric bypass: outcomes, complications, and predictors of response in 575 procedures. Gastrointest Endosc 2012;76:275-82.

32. Madan AK, Martinez JM, Khan KA, et al. Endoscopic sclerotherapy for dilated gastrojejunostomy after gastric bypass. J Laparoendosc Adv Surg Tech A 2010;20:235-7.

33. Baretta GA, Alhinho HC, Matias JE, et al. Argon plasma coagulation of gastrojejunal anastomosis for weight regain after gastric bypass. Obes Surg 2015;25:72-9.

34. Jirapinyo P, Kroner PT, Thompson CC. Sa2000 argon plasma coagulation in the treatment of weight regain following Roux-En-Y gastric bypass. Gastrointest Endosc 2017;85:AB270-1.
35. Thompson CC, Slattery J, Bundga ME, et al. Peroral endoscopic reduction of dilated gastrojejunal anastomosis after Roux-en-Y gastric bypass: a possible new option for patients with weight regain. Surg Endosc 2006;20:1744-8.

36. Ryou M, Mullady DK, Lautz DB, et al. Pilot study evaluating technica feasibility and early outcomes of second-generation endosurgical platform for treatment of weight regain after gastric bypass surgery. Surg Obes Relat Dis 2009;5:450-4.

37. Mullady DK, Lautz DB, Thompson CC. Treatment of weight regain after gastric bypass surgery when using a new endoscopic platform: initial experience and early outcomes (with video). Gastrointest Endosc 2009;70:440-4.

38. Jirapinyo P, Slattery J, Ryan MB, et al. Evaluation of an endoscopic suturing device for transoral outlet reduction in patients with weight regain following Roux-en-Y gastric bypass. Endoscopy 2013;45:532-6.

39. Patel LY, Lapin B, Brown CS, et al. Outcomes following 50 consecutive endoscopic gastrojejunal revisions for weight gain following Rouxen-Y gastric bypass: a comparison of endoscopic suturing techniques for stoma reduction. Surg Endosc 2017;31:2667-77.

40. Thompson CC, Chand B, Chen YK, et al. Endoscopic suturing for transoral outlet reduction increases weight loss after Roux-en-Y gastric bypass surgery. Gastroenterology 2013;145:129-37.

41. Kumar N, Thompson CC. Comparison of a superficial suturing device with a full-thickness suturing device for transoral outlet reduction (with videos). Gastrointest Endosc 2014;79:984.9.

42. Kumar N, Thompson CC. Transoral outlet reduction for weight regain after gastric bypass: long-term follow-up. Gastrointest Endosc 2016;83:776-9

43. Sarwer DB, von Sydow Green A, Vetter ML, et al. Behavior therapy for obesity: where are we now? Curr Opin Endocrinol Diabetes Obes 2009;16:347-52.

44. Mann JP, Jakes AD, Hayden JD, et al. Systematic review of definitions of failure in revisional bariatric surgery. Obes Surg 2015;25:571-4 